\title{
Density Stratification Effects on the Thermal Convection in a Rotating Spherical Shell
}

\author{
N. Nishikawa and K. Kusano \\ Graduate School of Advanced Sciences of Matter, Hiroshima University, \\ Higashi-Hiroshima 739-8526, Japan
}

\begin{abstract}
The density stratification effects on the thermal convection in a rotating spherical shell, which is the representative of the solar convection zone, are investigated by three dimensional numerical simulations. It is found that, the convection structure in the strongly stratified system is switched from parallel cells aligned to the rotation axis to zonal rolles dominated by the longitudinally averaged mode, as the Rayleigh number increases much larger than the stability threshold. Corresponding to this structural transition, the averaged kinetic helicity reverses the sign in each hemisphere (from negative to positive in the northern hemisphere). The results indicate that the density stratification is much important for the nonlinear convection process in the rotating spherical shell.
\end{abstract}

\section{Introduction}

Although the thermal convection is the most important physical process to understand the solar dynamo activity, the even basic properties have not been explained theoretically so far (Brummell et al. 1995). For instance, although the most of the previous simulations indicate that the longitudinally averaged kinetic helicity in the northern and the southern hemispheres could be negative and positive respectively, it is pointed out that such a helicity structure cannot construct the realistic butterfly diagram (Gilman \& Chabonneau 1999). Also the numerical simulations have not successfully created the realistic distribution of the mean angular velocity (Schou et al. 1998).

The discrepancy from the observations implies that the numerical models did not capture the important ingredient of the solar convection. Actually, some parameters adopted in the numerical simulation are far from the practical system, because of restriction in the computational capacity. In this study, we particularly consider the effects of the density stratification on the nonlinear convection based on the fully compressible numerical simulations.

\section{Models}

The simulation domain is a spherical shell rotating with the angular velocity $\boldsymbol{\Omega}=\Omega \mathbf{k}$, where the stress-free condition and the non-slip condition are applied on the top $\left(r=R_{t}\right)$ and the bottom $\left(r=R_{b}\right)$ boundaries, respectively. The temperature on those boundaries are fixed to $T_{t}$ and $T_{b}\left(T_{t}<T_{b}\right)$. The governing 

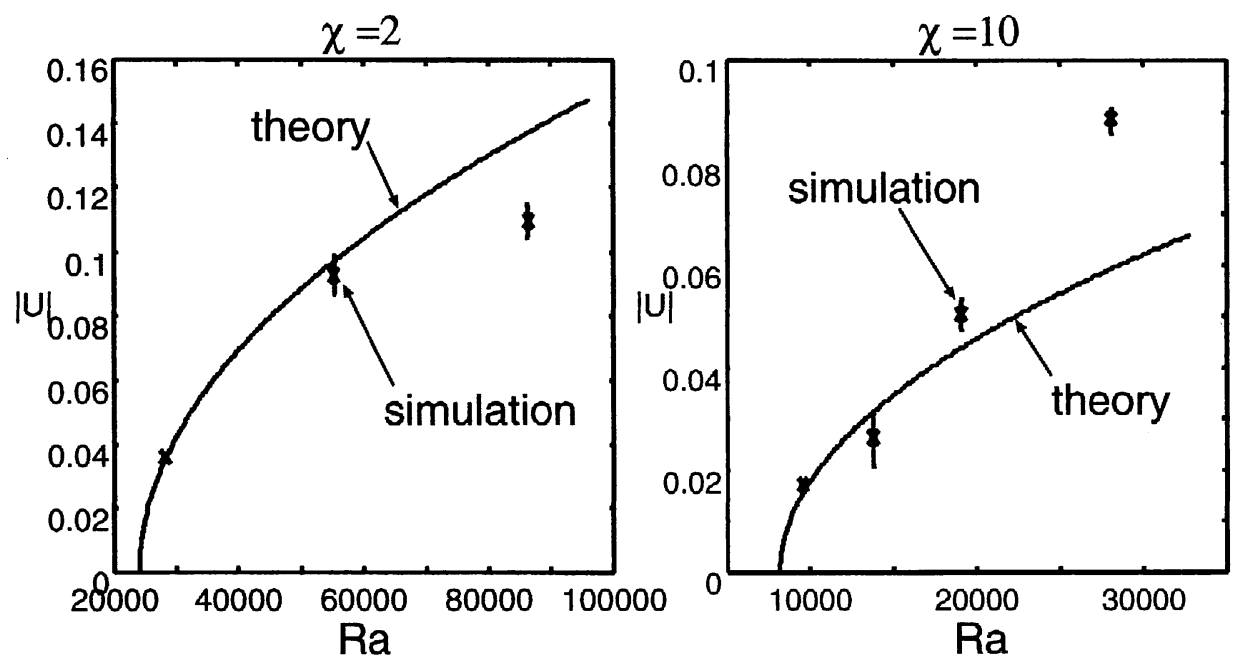

Figure 1. Average velocity in the final state is plotted as a function of Rayleigh number $R_{a}$ for $\chi=2$ (left) and 10 (right). The curve indicates the prediction from the weakly nonlinear theory.

equations are given by the followings:

$$
p=\rho T, \quad \frac{\partial \rho}{\partial t}+\nabla \cdot(\rho \mathbf{U})=0
$$

$\rho \frac{D \mathbf{U}}{D t}=-\nabla P-T_{a}^{\frac{1}{2}} P_{r a} C_{K}(\mathbf{k} \times \rho \mathbf{U})+P_{r a} C_{K}\left[\nabla^{2} \mathbf{U}+\frac{1}{3} \nabla(\nabla \cdot \mathbf{U})\right]-\beta(m+1) \frac{\rho}{r^{2}} \mathbf{r}$,

$$
\frac{D T}{D t}=-(\gamma-1) T \nabla \cdot \mathbf{U}+\frac{\gamma C_{K}}{\rho} \nabla^{2} T+\frac{2(\gamma-1) P_{r a} C_{K}}{\rho}\left(e_{i j} e_{i j}-\frac{1}{3}(\nabla \cdot \mathbf{U})^{2}\right),
$$

where the length and the time are normalized by the outer radius and the sound transit period, respectively.

The initial state is given by the hydrostatic condition, so that $T=\beta / r+$ $1-\beta$, and $\rho=T^{m}$ with the polytropic index $m$. Here, the Rayleigh number $R_{a}$ and the stratification ratio of the bottom and the top density $\chi=\rho_{b} / \rho_{t}$ are chosen as the parameters. The Pseudo-Spectral Fourier method is used not only for the longitudinal but also for the latitudinal derivatives (Fornberg 1996), and the radial derivative is approximated by the finite difference. The time integral is carried out using the Runge-Kutta-Gill method of the 4th order accuracy.

\section{Results and Discussions}

The calculations are performed for $\chi=2$ and 10, and for the Rayleigh number up to about three times larger than the stability threshold $R_{0}$. In any cases, a steady or a quasi-steady state is achieved. Figure 1 shows the dependency of the average velocity in the final state on the Rayleigh number. The results indicate 
(a) $\mathrm{Ra}=1.2 \mathrm{Ro}$

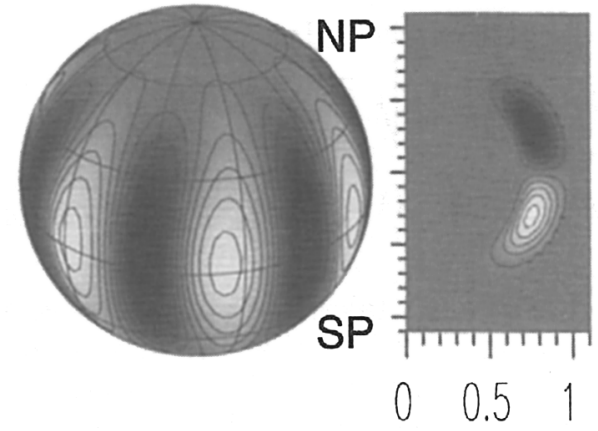

(b) $\mathrm{Ra}=3.4 \mathrm{Ro}$

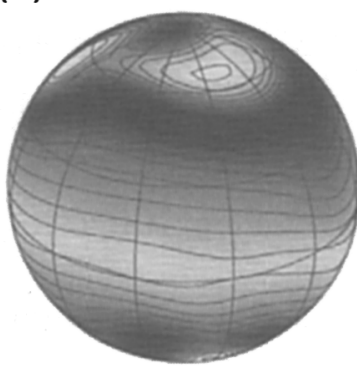

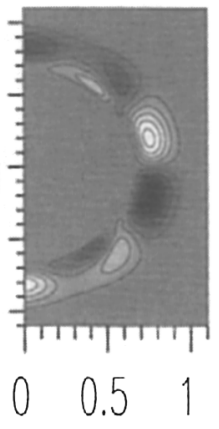

Figure 2. Radial velocity on a spherical surface and the averaged kinetic helicity on the meridional plane are drawn for (a) $R_{a}=1.2 R_{0}$ and (b) $R_{a}=3.4 R_{0}$, when $\chi=10$. White and black parts indicate the positive and the negative sign for each panel.

that, as the Rayleigh number increases, the convection in the strongly stratified system $(\chi=10)$ is more activated than the prediction of the weakly nonlinear theory $|U| \propto\left(R_{a}-R_{0}\right)^{1 / 2}$, although, in the case for $\chi=2$, the saturation level is lower than the theoretical prediction. It implies that a new type of solution arises when the density stratification is high enough.

Actually, as shown in Figs.2(a) and (b), in the strongly stratified case $(\chi=$ 10), the sectorial structure of the convection, which is a common feature with the previous simulations, is changed to the zonal structure which is dominated by the longitudinally averaged component, as the Rayleigh number increases. Furthermore, it clearly indicates that the longitudinally averaged kinetic helicity reverses the sigh in each hemisphere (from negative to positive in the northern hemisphere), corresponding to the structural conversion. In the weakly stratified case, however, the helicity reversal is not observed.

The appearance of the zonal convection for large Rayleigh number contrasts with the linear stability theory, because it predicts that the most unstable mode may shift to rather higher mode as Rayleigh number increases. They suggest not only that the linear theory is less reliable, but also that the density stratification is an important element when we consider the solar convection.

\section{References}

Brummell, N., Cattaneo, F. \& Toomre, J., 1995, Science, 269, 1370

Fornberg, B. 1996, A practical guide to pseudospectral methods (Cambridge University Press)

Gilman, P. A., \& Charbonneau, P. 1999, Magnetic Helicity in Space and Laboratory Plasmas, ed. Pevtsov et al., 75

Schou, J., et al. 1998, ApJ, 505, 390 